Michael Llamas, Maria Luisa Garo and Luca Giovanella*

\title{
Low free-T3 serum levels and prognosis of COVID-19: systematic review and meta-analysis
}

https://doi.org/10.1515/cclm-2021-0805

Received July 16, 2021; accepted July 30, 2021;

published online August 11, 2021

\section{Abstract}

Objectives: There is increasing interest regarding the relationship between serum levels of free triiodothyronine (fT3) and outcomes of COronaVIrus Disease-19 (COVID-19) patients. As several recent reports have described a worse prognosis in patients with low fT3 levels, we performed a meta-analysis to assess the prognostic role of fT3 serum levels in patients with COVID-19 as this information could be clinically relevant for the management of these patients. Methods: The methodology was registered in the International prospective register of systematic reviews (PROSPERO) database under the protocol number CRD42021260952. A systematic search was carried out on PubMed, Embase, Web of Science, and Scopus from May to June 2021 without time and language restrictions. The literature search strategy was based on the following keywords: (T3 OR fT3 OR triiodothyronine) AND (COVID-19) AND (prognosis OR survival).

Results: The literature search identified 163 studies. Seven retrospective studies met the inclusion and exclusion criteria and were included in the meta-analysis. The included studies had a total of 1,183 patients. From the analysis of the included studies, lower fT3 serum levels were consistently observed in intensive care unit (ICU) than in non-ICU patients and in nonsurvivors than survivors, respectively.

Conclusions: Serum fT3 concentrations are significantly lower in patients with severe COVID-19 than in non-severely

\footnotetext{
${ }^{*}$ Corresponding author: Prof. Dr. med. Luca Giovanella, MD, PhD, Clinic for Nuclear Medicine and Competence Center for Thyroid Diseases, Imaging Institute of Southern Switzerland, Ente Ospedaliero Cantonale, Via A. Gallino 12, Bellinzona 6500, Switzerland; and Clinic for Nuclear Medicine and Thyroid Centre, University Hospital of Zürich, Zürich, Switzerland, Phone: +4191811 86 72, Fax: +41 9181185 20, E-mail: luca.giovanella@eoc.ch. https://orcid.org/0000-0003-0230-0974

Michael Llamas, Intensive Care Unit, Locarno Regional Hospital, Ente Ospedaliero Cantonale, Locarno, Switzerland

Maria Luisa Garo, Mathsly Research, Brescia and Vibo Valentia, Italy
}

ill patients and predict all-cause mortality of patients with severe COVID-19. Accordingly, fT3 may become a simple tool for stratified management of patients with severe COVID-19.

Keywords: COronaVIrus Disease-19 (COVID-19); cytokines; euthyroid sick syndrome; free triiodothyronine; intensive care unit.

\section{Introduction}

The COronaVIrus Disease-19 (COVID-19) is an infectious disease caused by the enveloped RNA $\beta$-severe acute respiratory syndrome-coronavirus-2 (SARS-CoV-2). It may have a heterogeneous clinical presentation that varies from asymptomatic disease to acute respiratory distress syndrome (ARDS) and multi-organ failure. Since the pandemic outbreak, multiple studies have documented impairment of thyroid function with variable prevalence and severity in COVID-19 patients: abnormal thyroid function tests (thyroid stimulating hormone [TSH] and/or thyroid hormones) are frequently reported [1]. Three main mechanisms account for thyroid function perturbations in COVID-19 patients: (1) a direct viral effect on thyroid cells; (2) an indirect effect of systemic inflammatory immune response; and (3) the socalled euthyroid sick syndrome (ESS), a nonspecific adaptive mechanism for illness and an indirect marker of disease severity in various condition rather than a true thyroid dysfunction [2]. Interestingly, patients with COVID-19 may be affected by a combination of the above-mentioned pathways, i.e., the direct and indirect effects on target cells (thyroid, pituitary, and hypothalamus) and ESS may intertwine and give paradoxical results of hormonal status. For example, a combination of destructive thyroiditis and ESS may result in TSH suppression and increased free thyroxine (fT4) but decreased free triiodothyronine (fT3), defined as "low-T3 toxicosis" [2-4]. Moreover, recent studies suggested that ESS is the most common thyroid dysfunction observed in COVID-19 patients as a result of central (TSH decrease) and peripheric (reduced fT4 to fT3 conversion) effects of the cytokines storm [5-7]. Significantly lower serum concentrations of fT3 were found in patients with severe COVID-19 compared to non-severely ill patients; additionally, fT3 levels 
independently predicted all-cause mortality of patients with severe COVID-19, while no significant relationships were found between all-cause mortality and TSH, fT4, and fT3/fT4 ratio, respectively $[5,6,8]$. On these bases, fT3 was proposed as a candidate prognostic biomarker for stratified management of patients with severe COVID-19 [8].

This meta-analysis aimed to assess the prognostic role of fT3 serum levels in patients with COVID-19. Specifically, we aimed to address two clinical questions:

(1) Are serum fT3 levels predictive of clinical deterioration of COVID-19 patients requiring management in intensive care units (ICU)?

(2) Are serum levels of fT3 predictive of all-cause mortality in COVID-19 patients managed in ICUs?

\section{Materials and methods}

\section{Protocols and registration}

The material and methods were based on the Preferred Reporting Items for Systematic Reviews and Meta-Analyses (PRISMA) guidelines [9]. The methodology was previously registered in the International prospective register of systematic reviews (PROSPERO) database under the protocol number CRD42021260952.

\section{Eligibility criteria}

All original peer-reviewed research publications were considered. Eligible studies were selected according to the following criteria: (i) Research studies, cohort studies, prospective, and retrospective studies; (II) Studies involved adults admitted to hospital because of COVID-19 diagnosis; (III) studies in which fT3 serum levels of different groups (ICU vs. non-ICU, survivor vs. non-survivor) were listed in the form of mean \pm SD or median with interquartile range (IQR). Studies that had considered patients with a history of thyroid disorder or patients treated with anti-thyroid drugs and/or thyroid hormone and/or medications with potential impact on thyroid function, including systemic steroids, amiodarone, heparin, and dopamine were excluded.

\section{Information sources and search strategy}

A systematic search was carried out on PubMed, Embase, Web of Science, and Scopus from May to June 2021 without time and language restrictions. The literature search strategy was based on the following keywords: (T3 OR FT3 OR triiodothyronine)
AND (COVID-19) AND (prognosis OR survival). Additionally, hand searches were performed to identify articles other than those found in the electronic databases. A further hand search of the citation lists of the included studies was performed. Grey literature was searched using the Open Grey database (www. opengrey.eu) applied the previous search strategy. The first (title/abstract screening) and second (full-text assessment) steps of the search process were performed by two independent reviewers (LG and MG), and any disagreement was discussed until a decision was made by consensus.

\section{Study selection}

The complete list of articles obtained through the systematic search was screened to remove duplicates and exclude not eligible articles. The potentially relevant articles to answer the research question were screening by reading titles and abstracts. The eligible studies were independently selected by two reviewers (LG and MG). Full-texts of the remaining potentially relevant articles, those that met the inclusion and exclusion criteria, were retrieved. The final eligibility of each study was assessed, and the reasons for exclusion were recorded. Two authors (LG and MG) executed the definitive article selection. When there was disagreement, a third experienced reviewer (ML) was consulted to achieve a consensus.

\section{Data extraction}

The complete list of articles obtained by the systematic search was screened first to remove the duplicated and then to select the potentially relevant articles identified by titles that answered the research question. After, abstracts screening was conducted. The eligible studies were selected independently by two reviewers (LG and MG). In case of disagreement, it was resolved by discussion. From the remaining potentially relevant articles, full-text reading was done to select the articles that met the inclusion and exclusion criteria. Exclusion reasons were recorded. Data extraction was organized in a table that included the following information:

1) Study characteristics: first author, year, country, observation period;

2) Sample size and patients' characteristics (sex and age);

3) fT3 reference range and diagnostic tool;

4) COVID-19 severity assessment tool;

5) ICU admission criteria;

6) Number of patients admitted and non-admitted in ICU;

7) Number of patients survived and non-survived; 
8) Number of survived and non-survived patients according to their fT3 levels (lower than the low reference limit (LRL) vs. within the reference range (RR));

9) fT3 values (mean or median, standard deviation, IQR, or first and third quartile) for ICU and non-ICU patients and survived or non-survived patients.

No numerical information was extracted from the figures reported in the study publications.

\section{Quality assessment}

Two authors independently assessed the risk of bias of included studies using the NIH quality assessment tool for observational cohorts (https://www.nhlbi.nih.gov/ health-topics/study-quality-assessment-tools). The tool comprises 14 criteria; the overall assessment is defined as good, fair, and poor. Possible disagreements were resolved by discussion and consensus among all authors.

\section{Statistical analysis}

Continuous data were synthesized using mean difference (MD) with standard deviation from each study to calculate the average MD with 95\% confidence intervals (CIs). When continuous data were presented as medians and range, we applied Wan's formula to estimate the relative means and standard deviations [10]. Quantitative data were summarized through random-effect models described by DerSimonian and Laird [11] (first research question). The risk ratio was summarized through fixed-effects with the Mantel-Haenszel method (second research question). Heterogeneity was assessed using Cochrane Q-test and the $\mathrm{I}^{2}$ statistic, where a p-value $<0.05$ was taken to indicate statistically significant heterogeneity [12]. According to the Cochrane Handbook for Systematic Reviews of Interventions, the ranges of interpretation for $\mathrm{I}^{2}$ are as follows: $0-40 \%$ may be unimportant; $30-60 \%$ may represent moderate heterogeneity; 50-90\% may represent substantial heterogeneity, and $75-100 \%$ may have considerable

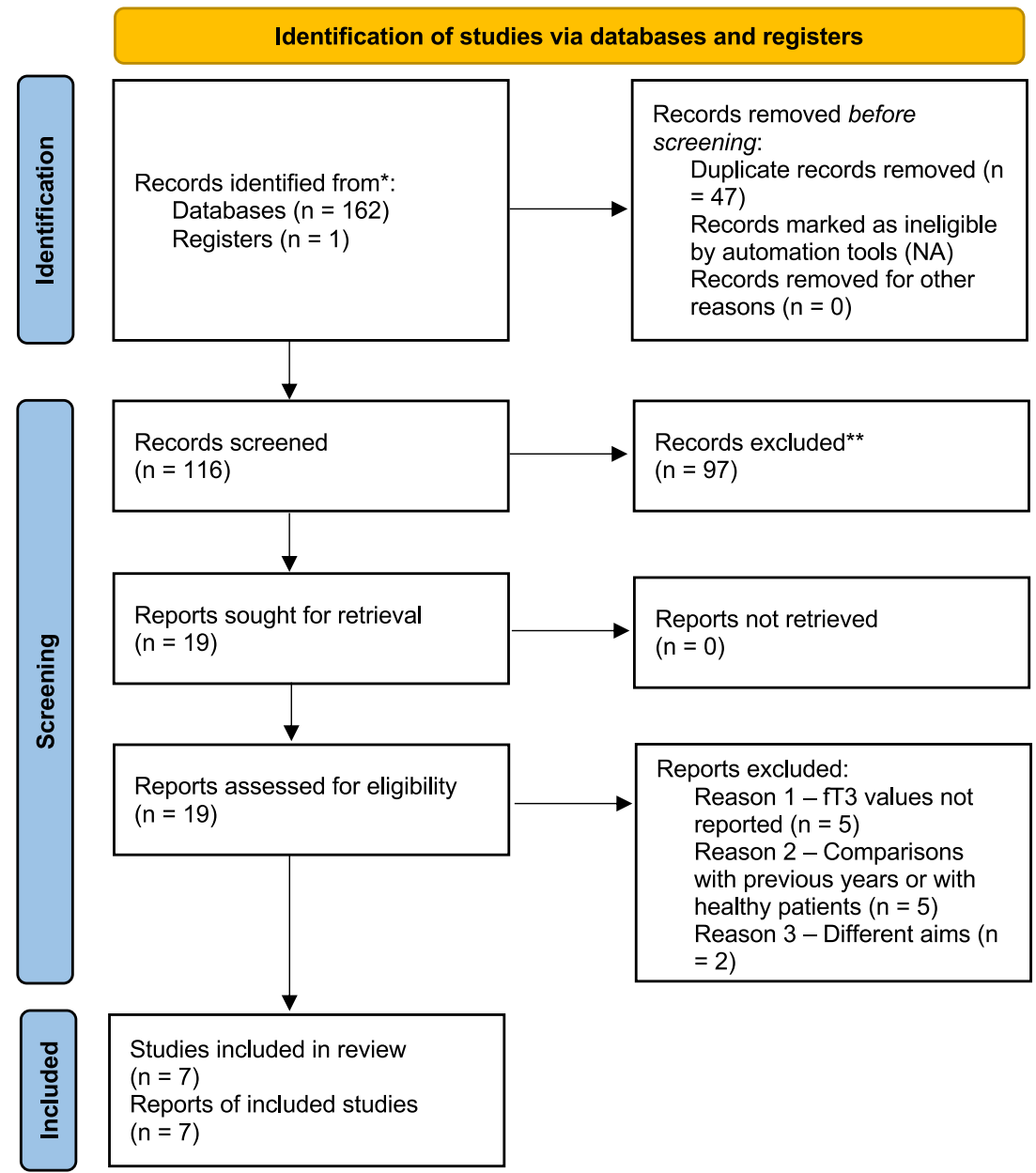

Figure 1: Preferred Reporting Items for Systematic Reviews and Meta-Analyses (PRISMA) flow-chart. 


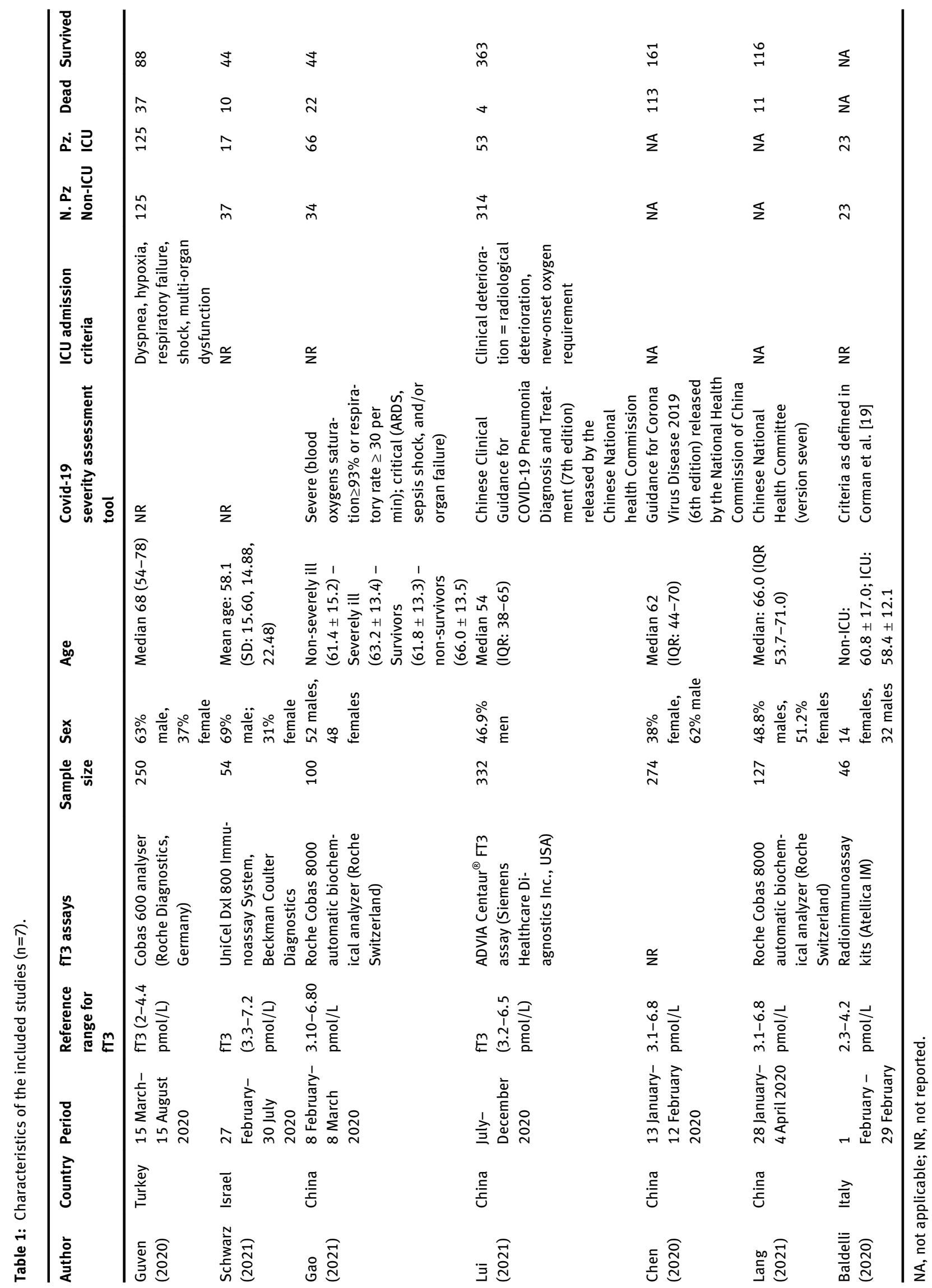


heterogeneity. We took values above $30 \%$ to indicate moderate heterogeneity. We had planned to analyze heterogeneity through subgroup analyses, but given the total of the included study, we did not perform this deeper analysis. Publication bias was not assessed according to 13.3.5.4. Recommendations on testing for funnel plot asymmetry [13]. A sensitivity analysis was performed to examine whether overall findings were robust to the chose analysis method. All analyses were conducted using STATA (17.0; StataCorp., College Station, TX, USA).

\section{Results}

\section{Search results and studies characteristics}

The literature search identified 163 studies. As shown in Figure 1, seven retrospective studies met the inclusion and exclusion criteria and were included in the metaanalysis [6, 8, 14-18]. Reasons for exclusion were also reported in Figure 1. The included studies had a total of 1,183 patients. The details of the included studies are reported in Table 1.

\section{Risk of bias assessment}

All studies were fair as assessed by the NIH tool. Details about the definition of assessment criteria and scores for criteria are reported in Table 2 . We noted that sample size justification and power description were not considered in all included studies. Some concerns were present in all studies about the possible role of the confounding variables. Five studies $[8,14-16,18]$ had no information about the participation rate of eligible persons.

\section{Meta-analysis}

The meta-analysis included seven studies published in 2020 and 2021 with a total of 1,183 COVID-19 hospitalized patients. Four studies were conducted in China, one in Israel, one in Italy, and one in Turkey. In total, 284 patients (24\%) were admitted to ICU. One hundred and ninety-three patients (16.3\%) died during hospitalization. The median age ranged from 54 [15] to 68 [14] years. A greater prevalence of males emerged in almost all studies but for Lui et al. [15]. COVID-19 severity was assessed using Chinese Clinical Guidance for COVID-19 Pneumonia Diagnosis and Treatment in two studies $[15,17]$. In Gao et al. COVID-19 severity was assessed according to the patient's clinical

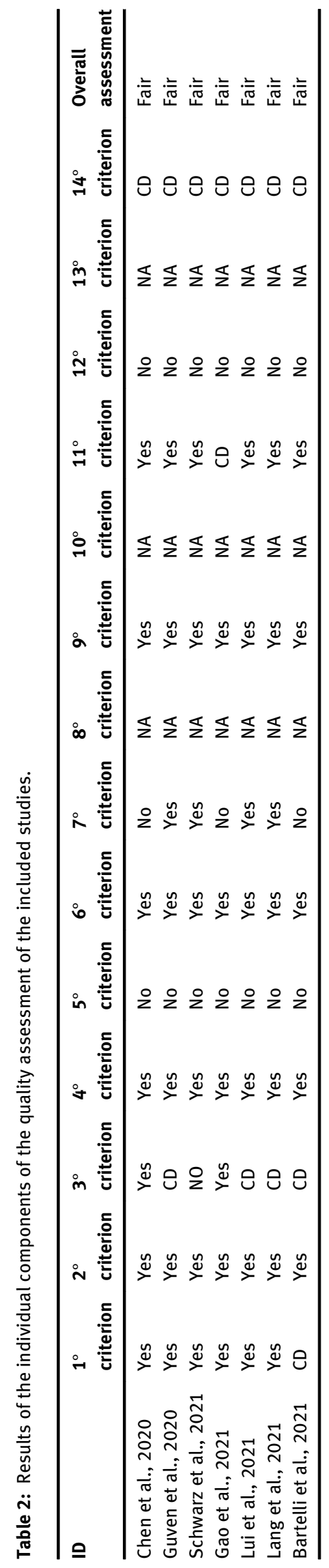




\begin{tabular}{|c|c|c|c|c|c|c|c|c|}
\hline \multirow[b]{2}{*}{ Study } & \multicolumn{3}{|c|}{ ICU patients } & \multicolumn{3}{|c|}{ non-ICU patients } & \multirow{2}{*}{$\begin{array}{l}\text { Hedges's g } \\
\text { with } 95 \% \mathrm{Cl}\end{array}$} & \multirow{2}{*}{$\begin{array}{c}\text { Weight } \\
(\%)\end{array}$} \\
\hline & $\mathrm{N}$ & Mean & SD & $\mathrm{N}$ & Mean & SD & & \\
\hline Guven, 2020 & 125 & 1.84 & .86 & 125 & 2.54 & .61 & $-0.94[-1.20,-0.68]$ & 44.50 \\
\hline Baldelli, 2021 & 23 & 1.77 & .68 & 23 & 2.4 & .75 & $-0.86[-1.46,-0.27]$ & 8.53 \\
\hline Gao, 2021 & 66 & 3.41 & .9 & 34 & 4.4 & .88 & $-1.10[-1.54,-0.66]$ & 15.74 \\
\hline Lui, 2021 & 48 & 3.63 & .67 & 284 & 4.2 & .74 & $-0.78[-1.09,-0.47]$ & 31.23 \\
\hline Overall & & & & & & & $-0.91[-1.08,-0.73]$ & \\
\hline
\end{tabular}

Heterogeneity: $\mathrm{T}^{2}=0.00, \mathrm{l}^{2}=0.00 \%, \mathrm{H}^{2}=1.00$

Test of $\theta_{i}=\theta_{j}: Q(3)=1.47, p=0.69$

Test of $\theta=0: z=-10.23, p=0.00$

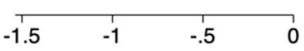

Figure 2: Forest plot for all studies comparing fT3 serum levels between ICU and non-ICU patients $(\mathrm{n}=4)$.

DerSimonian-Laird Random-effect model. Weighted $\mathrm{MD}=-0.91(95 \% \mathrm{Cl}-1.08 ;-0.73)$. Number of ICU and non-ICU patients, mean and standard deviation for fT3 serum levels for both groups. Hedges's g with $95 \% \mathrm{Cl}$ and weight have been reported.

\begin{tabular}{|c|c|c|c|c|c|c|c|c|}
\hline \multirow[b]{2}{*}{ Study } & \multicolumn{3}{|c|}{ Non-Survivors } & \multicolumn{3}{|c|}{ Survivors } & \multirow{2}{*}{$\begin{array}{l}\text { Hedges's g } \\
\text { with } 95 \% \mathrm{Cl}\end{array}$} & \multirow{2}{*}{$\begin{array}{c}\text { Weight } \\
(\%)\end{array}$} \\
\hline & $\mathrm{N}$ & Mean & SD & $\mathrm{N}$ & Mean & SD & & \\
\hline Chen, 2020 & 113 & 2.8 & .45 & 161 & 4.3 & .82 & $-2.16\left[\begin{array}{ll}-2.46, & -1.86\end{array}\right]$ & 21.74 \\
\hline Guven, 2020 & 37 & 1.5 & .63 & 88 & 1.98 & .93 & $-0.56\left[\begin{array}{ll}-0.95, & -0.17]\end{array}\right.$ & 21.16 \\
\hline Gao, 2021 & 22 & 2.76 & .49 & 44 & 3.73 & .88 & $-1.24[-1.79,-0.69]$ & 19.86 \\
\hline Lang, 2021 & 11 & 2.93 & .25 & 116 & 4.13 & .9 & $-1.38[-2.01,-0.74]$ & 19.03 \\
\hline Schwarz, 2021 & 10 & 3.45 & .77 & 44 & 4.65 & .93 & $-1.31[-2.03,-0.59]$ & 18.22 \\
\hline Overall & & & & & & & $-1.33[-2.03,-0.64]$ & \\
\hline
\end{tabular}

Heterogeneity: $\tau^{2}=0.55, l^{2}=90.58 \%, H^{2}=10.62$

Test of $\theta_{1}=\theta_{i}: Q(4)=42.47, p=0.00$

Test of $\theta=0: z=-3.76, p=0.00$

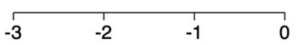

Figure 3: Forest plot for all studies comparing fT3 serum levels between dead and survivors $(n=5)$.

DerSimonian-Laird Random-effect model. Weighted $\mathrm{MD}=-1.33(95 \% \mathrm{Cl}-2.03 ;-0.64)$. Number of Survived and non-Survived patients, mean and standard deviation for fT3 serum levels for both groups. Hedges's $\mathrm{g}$ with $95 \% \mathrm{Cl}$ and weight have been reported.

\begin{tabular}{|c|c|c|c|c|c|c|c|}
\hline \multirow[b]{2}{*}{ Study } & \multicolumn{2}{|c|}{$<\mathrm{TT} 3 \mathrm{LRL}$} & \multicolumn{2}{|c|}{$\geq \pi \mathrm{L}$ LRL } & & \multirow{2}{*}{$\begin{array}{c}\text { Risk ratio } \\
\text { with } 95 \% \mathrm{Cl}\end{array}$} & \multirow{2}{*}{$\begin{array}{l}\text { Weight } \\
(\%)\end{array}$} \\
\hline & Non-Survivors & Survivors & Non-Survivors & Survivors & & & \\
\hline Schwarz (2021) - RR* $4.1-4.8$ pmol/ & 8 & 12 & 2 & 32 & $=$ & $5.67[1.32,24.37]$ & 21.42 \\
\hline Gao (2021) - RR: 3.1-6.8 pmo//L & 18 & 8 & 4 & 36 & - & $6.92[2.64,18.16]$ & 49.01 \\
\hline Lang (2021) - RR: 3.1-6.8 pmo//L & 8 & 13 & 3 & 103 & - & $13.46[3.89,46.59]$ & 29.57 \\
\hline
\end{tabular}

Heterogeneity: $\mathrm{T}^{2}=0.00, \mathrm{I}^{2}=0.00 \%, \mathrm{H}^{2}=1.00$

Test of $\theta_{i}=\theta_{j}: Q(2)=0.97, p=0.61$

Test of $\theta=0: t(2)=8.68, p=0.01$

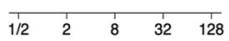

Figure 4: Forest plot for all studies comparing number of survived or nonsurvived patients with low and normal fT3 serum levels $(n=3)$.

Mantel-Haenszel fixed-effects model. Risk ratio $=8.07(95 \% \mathrm{Cl} 2.87 ; 22.72)$. Number of survived and non-survived patients according to the fT3 serum levels (lower than the normal reference range or within the normal reference range).

Table 3: Sensitivity analysis.

\begin{tabular}{|c|c|c|c|c|c|c|c|}
\hline \multicolumn{2}{|l|}{ ICU vs. no-ICU } & \multicolumn{2}{|c|}{ Survivors vs. no-survivors } & \multicolumn{4}{|c|}{ Below fT3 NR vs. fT3 NR } \\
\hline Fixed & Random & Fixed & Random & Fixed & Random & $\mathbf{R R}$ & OR \\
\hline $9=-0.91$ & $\vartheta=-0.91$ & $\vartheta=-1.48$ & $\vartheta=-1.34$ & $\vartheta=8.04$ & $\vartheta=8.39$ & $\vartheta=8.04$ & $\vartheta=17.02$ \\
\hline$[-1.08 ;-0.73]$ & {$[-1.08 ;-0.73]$} & {$[-1.68 ;-1.28]$} & {$[-1.89 ;-0.78]$} & {$[-4.10 ;-15.78]$} & {$[4.28 ; 16.46]$} & {$[-4.10 ;-15.78]$} & {$[7,22 ; 40.12]$} \\
\hline Test of $९$ : & Test of $९$ : & Test of $७:$ & Test of $९:$ & Test of ९: & Test of ९: & Test of $9:$ & Test of ९: \\
\hline$z=-10.23$ & $z=-10.23$ & $z=-14.65$ & $z=-4.71$ & $z=-6.06$ & $z=6.18$ & $z=-6.06$ & $z=-6.48$ \\
\hline$p<0.001$ & $p<0.001$ & $p<0.001$ & $p<0.001$ & $p<0.001$ & $p<0.001$ & $p<0.001$ & $p<0.001$ \\
\hline Test of $Q: x^{2}(3)$ & Test of $Q: x^{2}(3)$ & Test of $Q: x^{2}(4)$ & Test of $Q: X^{2}(4)$ & Test of $Q: x^{2}(2)$ & Test of $Q: X^{2}(2)$ & Test of $Q: x^{2}(2)$ & Test of $Q: x^{2}(2)$ \\
\hline$=1.47 ; p=0.69$ & $=1.47 ; p=0.69$ & $=42.47 ; p=0.00$ & $=42.47 ; p=0.00$ & $=0.81 ; p=0.67$ & $=0.79 ; p=0.67$ & $=0.81 ; p=0.67$ & $=0.45 ; p=0.80$ \\
\hline
\end{tabular}

LRL, low reference limit; $R R$, reference range.

conditions independently from a specific guideline [6]. Two studies did not report the COVID-19 severity assessment tool, while Baldelli et al. [18] based Covid-19 diagnosis on diagnostic criteria as defined in Corman et al. [19]. ICU admission criteria were reported by two studies $[14,15]$. 


\section{Meta-analysis of outcome}

A total of four studies examined the fT3 serum levels between ICU and non-ICU patients [6, 14, 15, 18], five studies reported fT3 serum levels in survivors and nonsurvivors $[6,8,14,16,17]$, and three studies analyzed the number of survived and no-survived patients according to their fT3 serum levels at admission (LRL vs. RR) $[6,8,16]$. From the analysis of the four studies comparing fT3 levels between ICU $(n=262)$ and non-ICU $(n=466)$ patients, lower fT3 serum levels were observed in ICU patients than in nonICU patients (WMD $=-0.91,95 \%$ CI: -1.08 ; -0.73 ; test of 9 : $\mathrm{z}=-10.23, \mathrm{p}<0.001$, test of homogeneity: $\mathrm{Q}=\chi^{2}(3)=1.47$, $\mathrm{p}=0.69, \mathrm{I}^{2}=0.00 \%$ ) (Figure 2). Lower fT3 serum levels were also observed in non-survivors $(\mathrm{n}=193)$ than in survivors $(\mathrm{n}=453)(\mathrm{WMD}=-1.33,95 \% \mathrm{CI}:-2.03 ;-0.64$; test of 9 : $\mathrm{z}=-3.76, \mathrm{p}<0.001$; test of homogeneity: $\mathrm{Q}=\chi^{2}(4)=42.47$, $\mathrm{p}<0.001 ; \mathrm{I}^{2}=90.58 \%$ ) (Figure 3). For patients with fT3 serum levels lower than the LRL at admission, the risk ratio was 8.07 [95\%CI: $2.87 ; 22.72]$ (test of $9: \mathrm{t}(2)=8.68, \mathrm{p}=0.01$; test of homogeneity: $\mathrm{Q}=\chi^{2}(2)=0.97, \mathrm{p}=0.61 ; \mathrm{I}^{2}=0.00 \%$ ) (Figure 4). The sensitivity analysis showed no significant differences from the previously reported results (Table 3 ).

\section{Discussion}

This meta-analysis aimed to determine whether fT3 serum levels predict hospitalization in ICUs and mortality in COVID-19 patients. Overall, across included studies, serum fT3 levels were consistently lower in ICU and non-survived patients compared to non-ICU and survived ones, supporting the role of fT3 as a prognostic biomarker in COVID-19 patients. The course and severity of COVID-19 are related to the activity of several cytokines and the presence of a cytokines storm induced by the virus. Accordingly, the excessive inflammatory response triggered by SARS-CoV-2 infection is likely the reason that COVID-19 patients develop ARDS, multi-organ failure, or death [20]. ESS is recognized as a nonspecific adaptive mechanism for illness and an indirect marker of disease severity in various conditions, including hospitalization in the critical care setting [21]. The underlying mechanisms include multiple and complex alterations (i.e., inhibition of iodide uptake and organification, suppression of thyroglobulin synthesis and reduction of thyroid hormone secretion, inhibition of hypothalamus-pituitary-thyroid axis) mediated by cytokines with specific effects on the thyroid gland as IL- 6 and TNF-alpha [22, 23]. Considering that IL-6 and TNF-alpha are specifically involved in the SARS-CoV-2-related cytokines storm [24] their increase is likely the cause of decreased fT3 levels in severely ill COVID-19 patients. Notably, the rise in inflammatory cytokines and the related decrease in fT3 occurs before the clinical deterioration in patients with COVID-19 [25]. Accordingly, intensive treatment measures should be taken to reduce the risk of death for patients with lower fT3 concentrations.

This study has several limitations. First, only retrospective studies were available, and the patients series' size was limited in some cases. Being all enrolled studies performed in COVID-19 hospitals using similar criteria, however, significant detrimental effects on the level of evidence are not expected. Second, different fT3 assays were employed in the included studies, precluding a cumulative ROC analysis. Accordingly, assay-specific reference ranges were used to define patients with reduced fT3 reflecting the current clinical and laboratory daily life practice.

Finally, the fT3 determination in COVID-19 patients should be considered in the context of other already identified prognostic biomarkers (e.g., procalcitonin, neutrophil-to-lymphocyte ratio, Creatine Kinase) [26-31].

In facts, different laboratory biomarkers showing inflammation or organ-damage also confer increased risk of severe COVID-19 disease or death. Multivariable models may provide integrated informations but using multiple prognostic factors for outcome prediction is challenging, especially when considering the high demand for accurate risk stratification for patients with COVID-19. Moreover, low fT3 at presentation remained a robust predictor of mortality in multivariate analyses that included all other significant predictors $[8,16,32]$.

This is likely due to different pathways captured by inflammation and organ-damage biomarkers and fT3, the latter being an integrative biomarker for adaption and host response to COVID-19 infection [8].

\section{Conclusions}

In conclusion, fT3 concentrations are significantly lower in patients with severe COVID-19 than in non-severely ill patients and predict all-cause mortality of patients with severe COVID-19. Thus, basing on our data, fT3 may become a simple tool for stratified management of patients with severe COVID-19.

Research funding: None declared.

Author contributions: All authors have accepted responsibility for the entire content of this submitted manuscript and approved its submission.

Competing interests: L.G. is a member of Roche Diagnostics Advisory Board and has received research grants from Roche Diagnostics and speaker honoraria from Roche Diagnostics and ThermoFisher BRAHMS. Other authors disclose no conflicts of interest. 
Informed consent: Not applicable. Ethical approval: Not applicable.

\section{References}

1. Giovanella L, Ruggeri RM, Ovčariček PP, Campenni A, Treglia G, Deandreis $D$. Prevalence of thyroid dysfunction in patients with COVID-19: a systematic review. Clin Transl Imag 2021;9: 233-40.

2. Giovanella L, Ruggeri RM, Petranović Ovčariček P, Campenni A, Treglia G, Deandreis D. SARS-CoV-2-related thyroid disorders: a synopsis for nuclear medicine thyroidologists. Eur J Nucl Med Mol Imag 2021;48:1719-23.

3. Muller I, Cannavaro D, Dazzi D, Covelli D, Mantovani G, Muscatello A, et al. SARS-CoV-2-related atypical thyroiditis. Lancet Diabetes Endocrinol 2020;8:739-41.

4. Lania A, Sandri MT, Cellini M, Mirani M, Lavezzi E, Mazziotti G. Thyrotoxicosis in patients with COVID-19: the THYRCOV study. Eur J Endocrinol 2020;183:381-7.

5. Khoo B, Tan T, Clarke SA, Mills EG, Patel B, Modi M, et al. Thyroid function before, during, and after COVID-19. J Clin Endocrinol Metab 2021;106:e803-11.

6. Gao W, Guo W, Guo Y, Shi M, Dong G, Wang G, et al. Thyroid hormone concentrations in severely or critically ill patients with COVID-19. J Endocrinol Invest 2021;44:1031-40.

7. Campi I, Bulgarelli I, Dubini A, Perego GB, Tortorici E, Torlasco C, et al. The spectrum of thyroid function tests during hospitalization for SARS COV-2 infection. Eur J Endocrinol 2021;184:699-709.

8. Schwarz Y, Percik R, Oberman B, Yaffe D, Zimlichman E, Tirosh A. Sick euthyroid syndrome on presentation of patients with COVID-19: a potential marker for disease severity. Endocr Pract 2021;27:101-9.

9. Moher D, Liberati A, Tetzlaff J, Altman DG. Preferred reporting items for systematic reviews and meta-analyses: the PRISMA statement. PLoS Med 2009;6:e1000097.

10. Wan X, Wang W, Liu J, Tong T. Estimating the sample mean and standard deviation from the sample size, median, range and/or interquartile range. BMC Med Res Methodol 2014;14:135.

11. DerSimonian R, Laird N. Meta-analysis in clinical trials revisited. Contemp Clin Trials 2015;45:139-45.

12. Higgins JPT, Thomas J, Chandler J, Cumpston M, Li T, Page MJ, Welch VA. Cochrane Handbook for Systematic Reviews of Interventions version 6.2 (updated February 2021). Cochrane; 2021. Available from: https://www.training.cochrane.org/ handbook (Accessed 31 Jul 2021).

13. Handbook.cochrane.org. Recommendations on testing for funnel plot asymmetry 2016. Available from: https://training.cochrane. org/handbook/current/chapter-13\#section-13-3-5-4 (Accessed 5 Jul 2021).

14. Güven $M$, Gültekin $H$. The prognostic impact of thyroid disorders on the clinical severity of COVID-19: results of single-centre pandemic hospital. Int J Clin Pract 2021;75:e14129.

15. Lui DTW, Lee CH, Chow WS, Lee ACH, Tam AR, Fong CHY, et al. Role of non-thyroidal illness syndrome in predicting adverse outcomes in COVID-19 patients predominantly of mild-tomoderate severity. Clin Endocrinol 2021:cen.14476. https://doi. org/10.1111/cen.14476.

16. Lang S, Liu Y, Qu X, Lu R, Fu W, Zhang W, et al. Association between thyroid function and prognosis of COVID-19: a retrospective observational study. Endocr Res 2021:1-8.

https://doi.org/10.1080/07435800.2021.1924770.

17. Chen T, Wu D, Chen H, Yan W, Yang D, Chen G, et al. Clinical characteristics of 113 deceased patients with coronavirus disease 2019: retrospective study. Br. Med. J. 2020;368:m1091.

18. Baldelli R, Nicastri E, Petrosillo N, Marchioni L, Gubbiotti A, Sperduti I, et al. Thyroid dysfunction in COVID-19 patients. J Endocrinol Invest 2021:1-5. https://doi.org/10.1007/s40618021-01599-0.

19. Corman VM, Landt O, Kaiser M, Molenkamp R, Meijer A, Chu DK, et al. Detection of 2019 novel coronavirus (2019-nCoV) by realtime RT-PCR. Euro Surveill 2020;25:2000045.

20. Coperchini F, Chiovato L, Croce L, Magri F, Rotondi M. The cytokine storm in COVID-19: an overview of the involvement of the chemokine/chemokine-receptor system. Cytokine Growth Factor Rev 2020;53:25-32.

21. Gutch M, Kumar S, Gupta KK. Prognostic value of thyroid profile in critical care condition. Indian J Endocrinol Metab 2018;22: 387-91.

22. Papanicolaou DA. Euthyroid sick syndrome and the role of cytokines. Rev Endocr Metab Disord 2000;1:43-8.

23. de Vries EM, Fliers E, Boelen A. The molecular basis of the nonthyroidal illness syndrome. J Endocrinol 2015;225:R67-81.

24. McGonagle D, Sharif K, O'Regan A, Bridgewood C. The role of cytokines including interleukin-6 in COVID-19 induced Pneumonia and macrophage activation syndrome-like disease. Autoimmun Rev 2020;19:102537.

25. Ragab D, Salah Eldin H, Taeimah M, Khattab R, Salem R. The COVID-19 cytokine storm; what we know so far. Front Immunol 2020;11:1446.

26. Izcovich A, Ragusa MA, Tortosa F, Lavena Marzio MA, Agnoletti C, Bengolea $A$, et al. Prognostic factors for severity and mortality in patients infected with COVID-19: a systematic review. PloS One 2020;15:e0241955.

27. Elshazli RM, Toraih EA, Elgaml A, El-Mowafy M, El-Mesery M, Amin $M N$, et al. Diagnostic and prognostic value of hematological and immunological markers in COVID-19 infection: a meta-analysis of 6320 patients. PloS One 2020;15:e0238160.

28. Khinda J, Janjua NZ, Cheng S, Heuvel ER, Bhatti P, Darvishian M. Association between markers of immune response at hospital admission and COVID-19 disease severity and mortality: a meta-analysis and meta-regression. J Med Virol 2021;93: 1078-98.

29. Alkhatip AAAMM, Kamel MG, Hamza MK, Farag EM, Yassin HM, Elayashy $M$, et al. The diagnostic and prognostic role of neutrophil-to-lymphocyte ratio in COVID-19: a systematic review and meta-analysis. Expert Rev Mol Diagn 2021;21:505-14.

30. Ulloque-Badaracco JR, Ivan Salas-Tello W, Al-kassab-Córdova A, Alarcón-Braga EA, Benites-Zapata VA, Maguiña JL, et al. Prognostic value of neutrophil-to-lymphocyte ratio in COVID-19 patients: a systematic review and meta-analysis. Int J Clin Pract 2021:e14596. https://doi.org/10.1111/ijcp.14596.

31. Akbar MR, Pranata R, Wibowo A, Lim MA, Sihite TA, Martha JW. The prognostic value of elevated creatine kinase to predict poor outcome in patients with COVID-19 - a systematic review and meta-analysis. Diabetes Metab Syndr Clin Res Rev 2021;15: 529-34.

32. Rothberger GD, Valestra PK, Knight K, Desai AK, Calixte R, Shapiro LE. Low free $T_{3}$ is associated with worse Outcomes in patients in the ICU requiring invasive mechanical ventilation. J Intensive Care Med 2021;36:313-8. 